\title{
Doit-on s'inspirer de la tarification à l'activité pour le financement des médicaments onéreux en établissements de santé ?
}

\author{
Jean-François Bussières, Mariève Simoncelli, Olivier Bourdon et Jean Lachaîne
}

\section{INTRODUCTION}

A u Canada, entre 1985 à 2005, l'ensemble des dépenses de santé a augmenté en moyenne de 6,5\% par année comparativement à 9,5\% pour les frais de médicaments. La part des médicaments au sein des dépenses totales de santé était estimée à près de $17 \%$ en 2007 pour un total de près de 30 milliards de dollars, ce qui la place au second rang des dépenses après les frais liés aux hôpitaux ${ }^{1}$. De plus, les dépenses en médicaments que recense l'Institut canadien d'information sur la santé n'incluent pas les dépenses en médicaments en hôpital, qui sont incluses dans le coût des hôpitaux. Le rapport 2007-2008 sur les pharmacies hospitalières canadiennes confirme cette croissance des coûts en médicaments ${ }^{2}$. Si les dépenses en médicaments continuent de croître à un rythme plus élevé que le reste des composantes du système de santé, une réflexion portant sur des approches permettant de favoriser une utilisation optimale des médicaments en établissement de santé s'impose. Cette dernière repose notamment sur une sélection appropriée des médicaments et sur le respect des règles d'utilisation et de remboursement établies par les décideurs, qui tiennent compte de données probantes, de l'état du patient et du rapport entre les coûts et les avantages.

Lobjectif de cet article est de présenter le modèle français de tarification à l'activité (T2A) et de gestion des médicaments onéreux de manière à susciter la réflexion quant à son applicabilité dans le contexte canadien de la gestion des médicaments en établissements de santé.

\section{LA TARIFICATION À L'ACTIVITÉ}

On définit la tarification à l'activité comme étant un système de financement des établissements de santé qui associe le paiement à l'activité réalisée, celle-ci étant définie par des épisodes de soins ${ }^{3}$. Il s'agit d'une méthode de paiement prospectif basé sur l'activité hospitalière plutôt que sur la dotation financière historique. L'activité hospitalière est définie à partir des diagnostics regroupés pour la gestion, aussi appelés groupes homogènes de malades plutôt qu'à partir des disciplines (p. ex. l'orthopédie) ou des services hospitaliers (p. ex. l'unité de soins de chirurgie).

De façon générale, la tarification à l'activité a été introduite pour augmenter l'activité hospitalière tout en permettant un rehaussement suffisant des bases budgétaires indexées basé sur la hausse des activités. En théorie, la tarification à l'activité peut contribuer à favoriser une meilleure transparence et une plus grande équité du mécanisme de financement des établissements dans la mesure où une certaine activité est rémunérée de la même façon dans chaque établissement. Elle vise à encourager l'efficience dans tous les établissements où le coût réel d'une activité donnée dépasse le tarif remboursé. Toutefois, la tarification à l'activité peut entraîner des conséquences non souhaitables, en incitant notamment les établissements à favoriser les activités rentables (c.-à-d. celles dont les coûts réels sont inférieurs aux tarifs) et à éliminer les activités qui ne le sont pas. La détermination de tarifs peut entraîner une réduction de la qualité des soins, entre autres par une réduction inappropriée de la durée de séjour ou de la nature de certains soins prodigués afin de satisfaire au tarif remboursés. En pharmacie, on peut présumer que, pour un tarif donné, on pourrait, par exemple, décider de supprimer certains soins pharmaceutiques (c.-à-d. des ressources en pharmaciens au sein d'une unité de soins) si les coûts réels associés à cette activité dépassent le tarif remboursé. De plus, la tarification à l'activité peut mener à un codage opportuniste des épisodes de soins afin d'apparier chaque épisode de soins au tarif le plus payant.

Plusieurs pays ont opté pour la tarification à l'activité au cours des dernières décennies, en commençant par les États- 
Unis (1983). Durant les années quatre-vingt-dix, plusieurs pays de l'Europe de l'Ouest ont intégré en partie ou en totalité la tarification à l'activité à leur système de financement (p. ex. Allemagne, Autriche, Belgique, Finlande, France, Irlande, Italie, Norvège, Portugal, Espagne, Suède), tout comme l'Australie d'ailleurs. Dans un rapport de l'Organisation mondiale de la santé publié en 2000 , on soulève le fait que la tarification à l'activité peut contribuer à améliorer la performance des systèmes de santé parce que ce modèle prend en considération la nature ainsi que la lourdeur des cas traités ${ }^{4}$. Plus récemment, la Grande-Bretagne et la France (2004) ont opté pour une réforme de leur système de santé, qui confere une large place à la tarification à l'activité. Bien que celle-ci ait été adoptée par plusieurs systèmes de santé, il est important de souligner que les paramètres de détermination des tarifs varient selon les pays.

Au Canada, "la majorité des dépenses publiques en santé est financée à même les fonds généraux des gouvernements provinciaux et fédéral, par les impôts sur les revenus des corporations et des particuliers $"$. Des discussions se poursuivent au niveau canadien (p. ex. Commission Kirby ${ }^{6}$ ) et au sein de plusieurs provinces quant à la pertinence d'introduire un financement basé sur les principes de la tarification à l'activitét ${ }^{\prime .8}$. De plus, des projets pilotes ont été menés (p. ex. Ontario Case Costing Initiative ${ }^{9}$ ), le financement des activités en chirurgie au Québec a été rehaussé en fonction de la prise en compte des volumes d'activité ${ }^{10}$, et des rapports ont recommandé l'implantation de ce nouveau mode de financement (p. ex. Comité sur la réévaluation du mode de budgétisation des centres hospitaliers de soins généraux et spécialisés - Rapport Bédard 2002 $2^{11}$. Malgré toutes ces initiatives, aucune province n’a encore opté pour ce mécanisme de financement à large échelle du réseau hospitaliser au Canada.

\section{LE MODÈLE FRANÇAIS}

Depuis 1983, le financement des hôpitaux du secteur public français était assuré par la dotation globale (c.-à-d. une enveloppe fermée accordée annuellement sur une base historique) alors que la tarification à la journée et à l'acte était appliquée aux hôpitaux du secteur privé12,13. L'une des problématiques qui a été associée à la dotation globale est l'insuffisance de financement qui en résulte pour les établissements dont le volume d'activités est élevé ou croissant ${ }^{12}$. La tarification à l'activité vise à uniformiser le financement entre les établissements de santé publics et privés ${ }^{14}$. Parmi les principes directeurs évoqués pour la mise en place de cette tarification en France, on note l'amélioration de la qualité et de la sécurité des prises en charge et des soins, la réduction des dépenses de santé, l'augmentation de l'autonomie des établissements, le maintien de la qualité et de l'équité dans l'accessibilité aux soins de santé, le développement de la responsabilité des acteurs hospitaliers et l'objectivation de l'affectation des ressources dans un contexte de raréfaction des ressources financières ${ }^{12,13-15}$.

La France a entrepris une réforme importante de son système de santé au cours de la dernière décennie. Ainsi, la tarification à l'activité a été progressivement implantée en France depuis 2005, et elle détermine le financement des établissements à $100 \%$ depuis 2008 (bien que la phase finale de son implantation ait été initialement prévue pour 2012) pour les programmes de médecine, de chirurgie, de gynécologieobstétrique et d'odontologie ${ }^{16}$. Chaque séjour de patients correspond à un groupe homogène de malades donné. Pour chaque groupe homogène de malades, on retrouve un groupe homogène de séjour (GHS) auquel l'application d'un tarif spécifique est prévue. Des ajustements aux groupes homogènes de séjour sont prévus lors de situations particulières (p. ex. séjours particulièrement longs, séjour en unités très spécialisées). On compte environ 700 groupes homogènes de séjour, et les forfaits qui y sont associés sont réévalués chaque année, notamment pour la prise en compte de l'impact de l'inflation ${ }^{12}$.

Le plan Hôpital 2007 vise à moderniser l'offre de soins par l'exercice d'une meilleure maitrise des dépenses de santé. Ce plan prévoit une refonte de la gouvernance hospitalière, comportant une plus grande concertation des directions hospitalières et une participation accrue des médecins à la prise de décisions stratégiques, le développement de pôles d'activités et la tarification à l'activitét ${ }^{12}$. Cette réorganisation hospitalière vise à favoriser une plus grande efficience et des économies par le regroupement des secteurs pouvant bénéficier d'une synergie de ressources. En pharmacie, la création de ces pôles a mené à des regroupements du département de pharmacie le plus souvent avec les laboratoires (p. ex. biochimie, pathologie, virologie) mais aussi avec d'autres secteurs. Il est encore trop tôt pour connaitre les effets de cette intégration des départements de pharmacie au sein de ces pôles. En France, les départements de pharmacie assurent la gestion de produits sanguins stables, de dispositifs médicaux stériles et de la stérilisation, et certains départements de pharmacie sont même responsables de certains dosages de médicaments et d'analyses toxicologiques. Plus récemment, le plan Hôpital 2012 a été adopté et vise, entre autres, l'organisation de schémas régionaux d'organisations sanitaires et l'accélération de la mise en œuvre de systèmes d'information hospitaliers incluant le processus de soins et le circuit du médicament ${ }^{17}$.

\section{LE CONTRAT DE BON USAGE}

En France, l'application de la tarification à l'activité requiert que chaque établissement ait signé un " contrat de bon usage " avec le directeur de l'Agence régionale de l'hospitalisation. Chaque contrat est adapté en fonction des besoins de l'établissement de santé concerné et est d'une durée de trois à cinq ans. Il définit les conditions d'utilisation et les modalités 
de remboursement qui sont établies en fonction d'indicateurs de suivi et de résultats. L'élaboration d'un rapport d'étape et d'un rapport final décrivant la progression effectuée permet l'évaluation de l'atteinte des objectifs. Le non-respect d'une ou de plusieurs clauses du contrat peut se traduire par des sanctions économiques, et il pourrait en résulter une diminution $\mathrm{du}$ financement des activités représentant jusqu'à $30 \%$ des dépenses engendrées ${ }^{12,15}$. Les principaux axes du contrat de bon usage sont les suivants ${ }^{15}:$ « chapitre I : amélioration et sécurisation du circuit du médicament et des produits et prestations; chapitre II : développement des pratiques pluridisciplinaires ou en réseau et respect des référentiels; chapitre III : mise en application des engagements spécifiques liés aux médicaments de la liste hors T2A, qui incluent des suivis de la consommation des médicaments par patient et par service et d'autres indicateurs devant être effectués par le département de pharmacie; chapitre IV : mise en place d'un dispositif d'audit interne et d'autoévaluations ciblées sur la prescription des médicaments de la liste hors T2A ».

Ainsi, les coûts de la plupart des médicaments prescrits lors d'un épisode de soins sont inclus à même le forfait des groupes homogènes de séjour. Comme certains médicaments et dispositifs médicaux implantables coûteux ne pouvaient pas être pris en compte adéquatement au sein des groupes homogènes de séjour, ils en ont été exclus. Pour le remboursement des médicaments exclus des groupes homogènes de séjour inscrits à la liste nationale, un montant additionnel aux prestations hospitalières de base associées à un groupe homogène de séjour donné est accordé aux établissements de santé $^{12}$.

\section{LA LISTE DES MÉDICAMENTS EXCLUS DES GROUPES HOMOGÈNES DE SÉJOUR}

La liste des médicaments exclus des groupes homogènes de séjour est établie par le ministère de la Santé français, et elle inclut des médicaments associés à un coût de traitement élevé ou qui se traduisent par "une hétérogénéité au niveau de la distribution des coûts par séjour ou par GHS ». Ainsi, un médicament sera remboursé lorsqu'il est prescrit pour une indication approuvée selon le référentiel de bon usage des médicaments exclus des groupes homogènes de séjour disponible sur le site de l'Agence française de sécurité sanitaire des produits de santé. Les référentiels de bon usage des médicaments exclus des groupes homogènes de séjour comportent : a) l'autorisation de mise en marché; b) les situations temporairement acceptables donnant lieu à des protocoles thérapeutiques temporaires et c) les situations non acceptables pour lesquelles le rapport risque-bénéfice est jugé défavorable selon l'état actuel des données scientifiques. Par ailleurs, des situations extérieures au référentiel, pour lesquelles le rapport risque-bénéfice ne peut être évalué en raison de l'insuffisance des données probantes disponibles, y sont également mentionnées. Ces situations font partie d'une zone grise. On rappelle que, exceptionnellement, lorsque le prescripteur ne se conforme pas aux référentiels de bon usage, il doit porter au dossier médical l'argumentation qui l'a conduit à sa prescription, en faisant référence à des travaux de groupes d'experts ou à des publications des revues internationales comportant un comité de lecture. La prescription de médicaments de cette liste, dans le cadre de maladies rares, peut faire l'objet d'une évaluation au cas par cas, après sollicitation de l'avis du centre de référence/compétence " labellisé » ${ }^{18}$.

Ainsi, la prescription de médicaments exclus des groupes homogènes de séjour est assortie d'une obligation pour le prescripteur de justifier par écrit que l'indication est conforme à l'autorisation de mise en marché ou à un protocole thérapeutique temporaire. Dans la négative, le prescripteur doit justifier l'utilisation d'un médicament exclu du référentiel, et il revient au pharmacien de l'établissement de santé d'en autoriser ou non l'usage sans connaître au préalable les conséquences sur le financement local. De plus, le directeur de l'établissement de santé doit transmettre avant le 15 octobre de chaque année un rapport d'étape annuel du Contrat de Bon Usage à l'Agence régionale de l'hospitalisation; celui-ci comprend le profil d'utilisation des médicaments exclus des groupes homogènes de séjour au sein de son établissement à des fins d'évaluation. Il s'agit d'une condition permettant de vérifier si l'usage de ces médicaments est conforme aux critères prédéfinis et de réévaluer ceux-ci le cas échéant. L'évaluation des données économiques permet d'une part, d'assurer l'efficience des médicaments inscrits à la liste et d'autre part, de permettre une meilleure prévision des dépenses à plus long terme ${ }^{19}$.

Les protocoles thérapeutiques temporaires sont définis par les organismes suivants : l'Agence française de sécurité sanitaire des produits de santé pour les médicaments, la Haute Autorité de santé pour les dispositifs médicaux et l'Institut national du cancer pour les anticancéreux et médicaments associés ${ }^{19}$. Il existe environ 400 protocoles pour lesquels des professionnels spécialisés du champ de pratique évalué ont défini l'indication, le schéma posologique ainsi que les éléments de suivi. Ces protocoles sont temporaires (durée maximale de quatre ans) et doivent être réévalués périodiquement pour la prise en compte de l'évolution des connaissances scientifiques ${ }^{19}$. Dans ce cadre, il revient aux établissements (en association avec la Commission du médicament et des dispositifs médicaux stériles) de définir les indications validées pour chaque médicament ciblé ainsi que les indications pour lesquelles les données probantes sont suffisantes, notamment celles provenant d'études de qualité élevée (essais cliniques contrôlés à répartition aléatoire). Au départ, la classification de ces indications a été réalisée par 37 groupes de travail formés par des experts (dont 12 pour les 
médicaments) qui sont des médecins et des pharmaciens spécialisés dans un champ thérapeutique donné. Pour conclure ce processus, l'Agence régionale de l'hospitalisation doit déterminer les indications reconnues pour lesquelles il existe une indication de paiement ${ }^{15}$.

De 2004 à 2006, on rapporte que la part des médicaments inscrits sur la liste d'exclusion des groupes homogènes de séjour est passée de 16 à $43 \%$ des dépenses de l'hôpital. La moitié de ces médicaments coûtent plus de 200 euros le format et 13 ont un prix supérieur à 2000 euros le format. En 2006, ces médicaments représentaient $61 \%$ de l'ensemble des dépenses de médicaments en France métropolitaine ${ }^{20}$.

Au 19 avril 2009, on compte 275 présentations de médicaments faisant partie de la liste des médicaments exclus des groupes homogènes de séjour (la tarification à l'activité), soit anticancéreux (110), médicaments associés à des anticancéreux (8), inhibiteurs du facteur de nécrose tumorale alpha (7), érythropoïétines (57), déficit enzymatique (8), hypertension artérielle pulmonaire (7), facteurs de coagulation (44), immunoglobulines (18), antifongiques (5) et autres (11). Par exemple, l'infliximab, en vertu de ce cadre de référence, est remboursé pour les indications reconnues à l'autorisation de mise en marché (p. ex. polyarthrite rhumatoïde, maladie de Crohn). L'utilisation de ce médicament est jugée temporairement acceptable pour six situations cliniques (c.-à-d. pyoderma grangrenosum de deux types, maladie de Still, maladie de Takayasu, polyangéite microscopique et Wegener, uvéite non infectieuse, etc.), non acceptable pour une situation clinique (c.-à-d. syndrome de Gougerot-Sjögren) et comportant une insuffisance de données pour six autres situations cliniques (c.-à-d.. maladie de Behcet autre que les uvéites, maladie de Horton, périartérite noueuse, sarcoïdose, onycho-pachydermo périostose psoriasique, maladie du greffon contre l'hôte).

\section{PERSPECTIVE CANADIENNE}

Au Canada, un fabricant obtient le droit de commercialiser un médicament après avoir obtenu un avis de conformité de Santé Canada. S'il désire que son produit soit remboursé par les régimes publics d'assurance-médicaments canadiens, il doit transmettre la documentation requise (c.-̀̀-d. monographie, études cliniques, etc.) au Programme commun d'évaluation des médicaments de l'Agence canadienne des médicaments et des technologies de la santé. Ce programme émet des recommandations relatives au contenu des listes de médicaments couverts par les régimes d'assurance-médicaments publics du Canada à l'exception du Québec. Toutefois, la décision définitive d'ajouter ou non un médicament à la liste revient à chacun des régimes provinciaux et territoriaux. Au Québec, ce rôle est assumé entièrement par le Conseil du médicament. Certains régimes établissent une liste distincte pour le milieu ambulatoire et le milieu hospitalier tandis que d'autres n'établissent qu'une seule liste pour les deux milieux de soins. Comme les règles régissant chacun de ces programmes et les listes des médicaments remboursés different d'une province et territoire à l'autre, ceci peut se traduire par des inégalités quant à l'accessibilité aux médicaments et particulièrement aux innovations coûteuses, tant en milieu ambulatoire qu'en milieu hospitalier. Il est reconnu que le système de remboursement dans certaines provinces et territoires est plus strict. À titre d'exemple, le taux de remboursement provincial pour le milieu ambulatoire en 2006 se situait entre 10\% (pour la ColombieBritannique et le Manitoba) et $80 \%$ (pour le Québec) du nombre total de nouveaux médicaments pour lesquels Santé Canada a émis un avis de conformitée ${ }^{21}$. Toutefois, il existe très peu de données comparant l'accessibilité aux médicaments en établissement de santé au Canada ${ }^{22,23}$. La boîte 1 résume les étapes menant à l'évaluation au niveau fédéral et provincial et à l'inscription d'un médicament aux listes des régimes publics d'assurance-médicaments provinciaux.

Peut-on s'inspirer du concept de tarification à l'activité et de gestion des médicaments exclus des groupes homogènes de séjour au niveau canadien? La santé étant principalement de compétence provinciale et territoriale, il revient à chaque province et territoire d'évaluer la faisabilité de l'implantation

\section{Boîte 1. Étapes menant à l'inscription d'un médicament aux listes de remboursement}

\begin{abstract}
Au niveau fédéral
Santé Canada émet un avis de conformité avec ou sans conditions sur présentation d'un nouveau médicament pour chaque indication demandée par le fabricant ${ }^{24}$

Émission par l'Agence canadienne des médicaments et des technologies de la santé (ACMTS) d'une recommandation définitive d'inscription au formulaire du Comité consultatif canadien d'expertise sur les médicaments sur demande d'un fabricant ${ }^{25}$

Le fabricant de médicaments brevetés fait rapport au Conseil d'examen du prix des médicaments brevetés (CEPMB) dans les 60 jours qui suivent la première vente. Le CEPMB vérifie que le prix de vente au « départ de l'usine » n'est pas excessif.
\end{abstract}

\section{Au niveau provincial et territorial}

Assurance-médicaments et pharmacie communautaire Les fabricants transmettent la documentation requise aux régimes d'assurance-médicaments publics (y compris les agences de cancer et l'initiative Joint Oncology Drug Review ${ }^{26}$ qui procèdent ou non à l'inscription d'un médicament en tenant compte de la recommandation de l'ACMTS.

Un médicament peut être inscrit avec ou sans conditions sur une liste de médicaments couverts pour une province donnée ou un territoire donné; certaines provinces et territoires ont une liste distincte pour les établissements de santé.

\section{Établissement de santé}

Les fabricants transmettent la documentation requise aux groupes d'achats et aux pharmaciens-chefs des établissements de santé qui procèdent à l'inscription d'un médicament en tenant compte de la recommandation de comités d'usagers, de comités régionaux (p. ex., Programme de gestion thérapeutique des médicaments), de comités de pharmacologie locaux, de médecins et de chefs de départements de pharmacie. 
d'une tarification à l'activité en santé. Au niveau des établissements de santé, il existe plusieurs barrières à ce changement de mode de budgétisation, notamment la nécessité d'adapter plusieurs systèmes d'information de gestion afin de pouvoir imputer les coûts par activité plutôt que par secteur de soins. Depuis quelques années, on applique la dixième édition de la classification internationale des maladies ${ }^{27}$, ce qui influence largement le codage des épisodes de soins et potentiellement la distribution des tarifs applicables à chaque établissement. On ne connaît pas encore les effets du changement de classification sur le profil de codification des établissements de santé.

En ce qui concerne la gestion des médicaments onéreux en établissement de santé, on observe déjà l'émergence d'autres choix de mécanismes de financement. Par exemple, plusieurs provinces ont mis à contribution leur agence du cancer afin d'établir des règles d'utilisations (c.-à-d. référentiels) avec ou sans conséquences sur le financement de chaque établissement. Il n'existe aucune donnée sur l'efficience de financements provenant de plusieurs sources (c.-à-d. une partie du financement des médicaments provient du ministère de la Santé et une autre de l'Agence du cancer pour certains médicaments anticancéreux conformes aux référentiels en place). Cependant, il nous apparaît peu souhaitable d'envisager un tel fractionnement qui entraîne la multiplication de tiers payeurs. Bien que ce fractionnement puisse établir un lien entre le payeur et celui qui établit des règles d'utilisation de médicaments pour une classe thérapeutique donnée, par exemple, il multiplie néanmoins les sources de financement et peut compliquer la gestion globale des médicaments. Dans l'exemple touchant la cancérologie, les patients recevant de la chimiothérapie prennent plusieurs autres classes de médicaments, comme des anti-infectieux, des facteurs de stimulation des colonies de granulocytes, des benzodiazépines, etc. Il nous apparaît préférable que l'ensemble du budget médicament demeure sous la responsabilité du chef du département de pharmacie pour assurer des décisions cohérentes dans leur ensemble, et non par groupes de patients, par classe thérapeutique ou de façon encore moins souhaitable, par médicament remboursé ou non. Par ailleurs, il nous apparaît très pertinent que des règles d'utilisation provinciale soient adoptées pour favoriser une pratique cohérente à l'échelle du réseau, ce qui donnerait de meilleurs indicateurs de prise de décision aux chefs de départements de pharmacie des établissements de santé.

Un autre exemple touche les maladies métaboliques héréditaires rares, où les gouvernements ont accepté, pour des cas pris individuellement et pour certains patients, de rembourser le coût du médicament onéreux même s'il est administré en traitement ambulatoire. Quelques régimes provinciaux réfléchissent aux modalités d'évaluation et de remboursement de ces médicaments. Au Québec, une circulaire administrative a été rédigée spécifiquement pour assurer le suivi et le financement de l'utilisation adéquate du bévacizumab $^{28}$. On imagine mal que ce mécanisme soit utilisé à nouveau pour d'autres médicaments sans qu’un cadre général de gestion des médicaments onéreux ne soit défini.

De façon générale, les chefs de départements de pharmacie et les comités de pharmacologie des établissements ont accepté d'utiliser des médicaments sans obtenir d'avis favorable des régimes provinciaux, compte tenu de la nature tertiaire de plusieurs établissements, du contexte clinique et de la gravité de certains cas réfractaires aux médicaments disponibles, de l'utilisation préalable de certains de ces médicaments dans le cadre d'études postcommercialisation ou du Programme d'accès spécial de Santé Canada, des délais d'évaluation et de décision pour leur ajout aux listes de médicaments couverts par les régimes publics et de l'absence de liens entre les listes de médicaments et le remboursement en dotation globale. Toutefois, la mise en marché de plusieurs médicaments onéreux ne permet plus aux établissements d'absorber les coûts additionnels grâce aux indexations consenties en dotation globale, même si le facteur d'indexation est plus élevé pour les médicaments que pour d'autres dépenses de santé.

À la lumière du modèle français, nous retenons les applications pratiques potentielles suivantes. Les recommandations proposées par l'Agence canadienne des médicaments et des technologies de la santé pourraient aussi être appliquées en établissements de santé par l'intermédiaire des comités de pharmacologie. Dans les cas où les données probantes sont insuffisantes, la rédaction de protocoles thérapeutiques temporaires à l'échelle d'une province ou d'une région (p. ex. les règles proposées par le programme de gestion thérapeutique des médicaments des cinq centres hospitaliers universitaires du Québec ${ }^{29}$ ) pourrait être effectuée pour déterminer la conformité de l'utilisation. Dans le cadre du déploiement du dossier santé électronique dans différentes provinces, il apparaît opportun de déterminer des champs obligatoires permettant la justification de la conformité au moment de la prescription de médicaments onéreux, tels que l'infliximab; l'intégration de ces champs dans les prescripteurs électroniques utilisés en établissement de santé devrait faire partie des données échangées en traitement ambulatoire pour assurer la continuité des soins et du remboursement entre les différents milieux de soins. Au Québec, les prescripteurs peuvent désormais indiquer un code de remboursement pour certains médicaments d'exception afin d'en établir la conformité et d'en faciliter la prescription et la saisie de l'indication. En attendant le déploiement de prescripteurs électroniques à l'échelle des établissements, rien n'interdit la mise en place, à petite échelle pour quelques médicaments, d'une gestion des indications codifiées et d'une saisie dans les progiciels pharmacie. 
Le tableau 1 présente une ventilation des médicaments par dénomination générique et forme posologique/teneur, faisant l'objet d'un statut d'exception pour le Québec. Ainsi, un nom générique peut être compté une ou plusieurs fois selon le nombre de formes/teneurs inscrites sur la liste. Des 302 médicaments mentionnés dans l'une des quatre listes, nous notons un nombre plus faible de médicaments faisant l'objet d'un statut d'exception en France par rapport au Québec, tant en pharmacie communautaire qu'en établissement de santé. De plus, il est important de noter qu'il existe des différences quant au contenu des listes, particulièrement quant aux facteurs de coagulation et aux immunoglobulines inscrits en France mais pas au Québec et quant aux formules nutritives et pansements inscrits au Québec mais pas en France. Ces produits sont gérés par des mécanismes différents en France ou au Québec selon le cas. Ces données laissent entendre qu'il est réaliste de mettre en place une codification des indications à titre de médicaments d'exception en établissement de santé afin d'assurer une prescription conforme et une description de l'indication à la saisie par le pharmacien. Une évaluation de la pertinence de la sélection des médicaments requérant un code doit toutefois être effectuée.

Au Canada, il n'existe pas de mécanisme centralisé de collecte de données d'utilisation des médicaments onéreux en établissement de santé. Toutefois, au cours des dernières années, des initiatives, comme le Service canadien de prescription et d'utilisation optimales des médicaments, ont été mises en place afin d'effectuer des examens systématiques de certaines classes thérapeutiques, telles que les inhibiteurs de la pompe à proton. Le Service canadien de prescription et d'utilisation optimales des médicaments est l'un des programmes de l'Agence canadienne des médicaments et des technologies de la santé, dont le mandat est de développer des outils visant une utilisation optimale des médicaments basée sur des données probantes ${ }^{30}$. En France, un observatoire des médicaments, des dispositifs médicaux et des innovations thérapeutiques a été établi afin de permettre l'analyse des pratiques en place dans les différentes régions et d'assurer une plus grande uniformité au sein de ce processus $^{31}$. Le modèle économique des médicaments et dispositifs médicaux implantables à l'extérieur des groupes homogènes de séjour avait comme objectif une diffusion de l'innovation et une égalité d'accès des patients quel que soit leur lieu de résidence. Une fois la diffusion réalisée et la place dans la thérapie établie, le coût du médicament devrait finalement être intégré aux coûts associés au groupe homogène de séjour. Laugmentation des dépenses liées aux médicaments exclus des groupes homogènes de séjour étant très importante, une mesure vient d'être introduite en 2009 afin d'en limiter la progression à $10 \%$, ce qui rend à nouveau le système contraignant ${ }^{32}$.

\section{Tableau 1. Répartition des médicaments ayant un remboursement lié à l'indication de façon contraignante}

\begin{tabular}{|c|c|c|c|c|c|}
\hline \multirow[b]{2}{*}{$\begin{array}{l}\text { Classe thérapeutique selon le } \\
\text { modèle français }\end{array}$} & \multicolumn{2}{|c|}{ France* } & \multicolumn{2}{|c|}{ Québect } & \multirow[b]{2}{*}{ Total } \\
\hline & Ambulatoire & Hospitalisé & Ambulatoire & Hospitalisé & \\
\hline Organisation & $\begin{array}{l}\text { Commission de } \\
\text { la transparence } \\
\text { (médicaments } \\
\text { d'exception) }\end{array}$ & $\begin{array}{l}\text { Agence française } \\
\text { de sécurité } \\
\text { sanitaire des } \\
\text { produits de santé } \\
\text { (médicaments }\end{array}$ & $\begin{array}{l}\text { Conseil du } \\
\text { médicament } \\
\text { (médicaments } \\
\text { d'exception) }\end{array}$ & $\begin{array}{l}\text { Conseil du } \\
\text { médicament } \\
\text { (médicaments } \\
\text { d'exception) }\end{array}$ & \\
\hline
\end{tabular}

\begin{tabular}{|c|c|c|c|c|c|}
\hline Anticancéreux & & 41 & 2 & 14 & 41 \\
\hline Antifongiques & & 3 & 1 & 1 & 3 \\
\hline Autres & & 11 & 2 & 3 & 11 \\
\hline Déficit enzymatique & & 9 & & & 9 \\
\hline Érythopoïétines & 5 & 5 & 2 & 2 & 5 \\
\hline Facteurs de la coagulation & & 14 & & & 14 \\
\hline Hypertension artérielle pulmonaire & & 3 & 2 & 2 & 3 \\
\hline Immunoglobulines & & 5 & & & 5 \\
\hline Inhibiteurs du TNF $\alpha$ & 2 & 3 & 3 & 3 & 3 \\
\hline Médicaments associés aux anticancéreux & & 8 & & 2 & 8 \\
\hline $\begin{array}{l}\text { Médicaments non repris dans la } \\
\text { liste hors GHS }\end{array}$ & 25 & & 171 & 139 & 200 \\
\hline$\overline{\text { Total }}$ & 32 & 102 & 183 & 166 & 302 \\
\hline
\end{tabular}




\section{CONCLUSION}

Plusieurs pays ont mis en place une budgétisation fondée sur la tarification à l'activité. En France, ce modèle a permis la mise en place d'un financement complémentaire des médicaments onéreux, dont l'utilisation est conforme à une règle d'utilisation. Au Canada, il apparaît nécessaire de déterminer des modalités d'évaluation et de gestion du remboursement des médicaments onéreux en établissement de santé, en dotation budgétaire globale ou, éventuellement, à l'activité. Le modèle français propose des pistes de solution pour la gestion des médicaments onéreux, qui devraient être évaluées dans le cadre d'une étude pilote de faisabilité. Pour la sauvegarde du principe d'égalité d'accès, cette étude devrait être menée par des centres universitaires et d'autres centres qui pourraient avoir accès à ces médicaments plus spécialisés.

\section{Références}

1. Dépenses en médicaments au Canada de 1985 à 2007. Ottawa (ON) : Institut canadien d'information sur la santé; 2008. Publié à http://secure.cihi.ca/cihiweb/products/Drug_Expenditure_in_ Canada_1985_2007_f.pdf. Consulté le 12 avril 2009.

2. Babich M, Bussières JF, Hall KW, Harding J, Johnson N, Lefebvre P, et coll., rédacteurs. Pharmacies hospitalières canadiennes rapport 2007-2008. Eli Lilly; 2008. Publié à www.lillyhospitalsurvey.ca/hpc2/content/ rep_2008_tocF.asp. Consulté le 22 avril 2009.

3. Or Z, Renaud T. Principes et enjeux de la tarification à l'activité à l'hôpital (T2A). Enseignements de la théorie économique et des expériences étrangères [document de travail]. Paris (France) : Institut de recherche et documentation en économie de la santé; 2009. Publié à www.irdes.fr/EspaceRecherche/DocumentsDeTravail/DT23Princip EnjeuxTarificActiviteHopital.pdf. Consulté le 12 avril 2009.

4. Rapport sur la santé dans le monde, 2000_pour un système de santé plus performant. Genève (Suisse): Organisation mondiale de la santé; 2009. Publié à www.who.int/whr/2000/fr/index.html. Consulté le 12 avril 2009.

5. Garantir l'accès : un défi d'equité, d'efficience et de qualité [document de consultation]. Québec (QC) : Ministère de la Santé et des Services Sociaux; 2006. Publié à http://publications.msss.gouv.qc.ca/acrobat/f/ documentation/2005/05-721-01.pdf. Consulté le 12 avril 2009.

6. Kirby MJL, président du comité. La santé des Canadiens-le rôle du gouvernement fédéral. Rapports intérimaires. Ottawa $(\mathrm{ON})$ : Le comité sénatorial permanent des Affaires sociales, de la science et de la technologie; 2000-2002. (Rapport Kirby).

7. Rich P. Time for a new approach to hospital funding? Ottawa (ON) : Association médicale canadienne; 2007. Publié à www.cma.ca/ index.cfm?ci_id=10041323\&la_id=1. Consulté le 18 avril 2009.

8. Collier R. Activity-based hospital funding: boon or boondoggle? CMAJ 2008;178(11):1407-1408.

9. Ontario Case Costing Initiative [site web]. Toronto $(\mathrm{ON})$ : Ontario Case Costing Initiative; 2009. Publié à www.occp.com/. Consulté le 19 avril 2009.

10. Règles de financement: production en chirurgie, 2007. Québec (QC) : Ministère de la Santé et des Services Sociaux; 2007.

11. La budgétisation et la performance financière des centres hospitaliers: rapport Bédard 2002. Québec (QC) : Ministère de la Santé et des Services Sociaux; 2002. Publié à http://msssa4.msss.gouv.qc.ca/fr/document/ publication.nsf/ed7acbc94b12630f852566de004c8587/a46c72fe10c0cbc 385256b9600553dec?OpenDocument. Consulté le 18 avril 2009.

12. Paubel P, Aoustin M. Médicaments et dispositifs dans le cadre de la tarification de l'activité. AMIPS info [Association des médecins des industries de produits de santé] 2005;70(4):64-72.
13. Andréoletti $\mathrm{C}$, et coll. Mission tarification à l'activité. La tarification des établissements de santé. Paris (France) : Gouvernement de la France; 2007. Publié à www.sante.gouv.fr/htm/dossiers/t2a/accueil.htm. Consulté le 12 avril 2009.

14. Aoustin M, Rieu C, Hauser S. T2A et dispositifs médicaux : nouvelles dispositions et perspectives tarifaires. AMIPS info [Association des médecins des industries de produits de santé] 2006;74:100-110.

15. Dode X, Fraslin B, Armoiry O, Faujour V, Aulagner G. Le contrat de bon usage du médicament: du concept à l'action, un itinéraire semé d'embûches. Gest Hosp 2005;450:707-16.

16. Claveranne JP, Pascal C. Tarification à l'activité et management hospitalier, vers de nouveaux défis. Oxymag 2006;19(86):19-22. Publié à www. emconsulte.com/article/84049. Consulté le 27 avril 2010.

17. Lancement du plan Hôpital 2012. Paris (France) : Gouvernement de la République française, Ministère de la Santé et des Sports; 2008. Publié à www.sante-sports.gouv.fr/actualite-presse/presse-sante/communiques/ lancement-du-plan-hopital-2012-au-coeur-modernisation-du-paysagehospitalier-francais.html. Consulté le 12 avril 2009.

18. Tarification à l'activité et médicaments hors du groupe homogène de séjour. Agence française de sécurité sanitaire des produits de la santé; 2009 Publié à www.Afssaps.fr/Dossiers-thematiques/Tarification-a-l-activiteT2A-medicaments/Accueil-T2A/\%28offset\%29/0. Consulté le 19 avril 2009.

19. Dumarcet N. Tarification à l'activité (T2A) et référentiels nationaux pour un bon usage des médicaments onéreux et innovants en médecine interne. Rev Med Interne 2008;29(2):84-86.

20. Clerc ME, Haury B, Rieu C. Études et résultats no 653 : Les médicaments hors tarification à l'activité dans les établissements de santé. Paris (France) : Gouvernement de la République françise; 2008. Publié à www.sante.gouv.fr/drees/etude-resultat/er-pdf/er653.pdf. Consulté le 31 octobre 2009.

21. Skinner BJ. Waiting for reimbursement of new medicines in Canada: It's time for a rethink. Pharmacoeconomics 2008;26(8):629-632.

22. Mittmann N, Knowles S. A survey of pharmacy and therapeutic committees across Canada: scope and responsibilities. Can J Clin Pharmacol 2009;16(1):e171-e177.

23. Shalansky SJ, Virk R, Ackman M, Jackevicius C, Kertland H, Tsuyuki R, et al. Access to new cardiovascular therapies in Canadian hospitals: a national survey of the formulary process. Can J Cardiol 2003;19(2): 173-179.

24. Avis de conformité. Ottawa $(\mathrm{ON})$ : Santé Canada; 2005. Publié à www.hc-sc.gc.ca/dhp-mps/prodpharma/notices-avis/index-fra.php. Consulté le 27 avril 2010.

25. Le processus PCEM. Ottawa $(\mathrm{ON})$ : Agence canadienne des medicaments et des technologies de la santé; 2010. Publié à www.cadth.ca/index.php/ fr/cdr/process. Consulté le 27 avril 2010.

26. Joint oncology drug review. Toronto $(\mathrm{ON})$ : Ministère de la Santé et des Soins de Longue Durée; 2009. Publié à www.health.gov.on.ca/ english/providers/program/drugs/drug_submissions/inter_oncology_drugs. html. Consulté le 22 avril 2009.

27. [Classification internationale des maladies, dixième version (CIM-10)]. Genève (Suisse) : Organisation mondiale de la santé; 2007. Publié à http://apps.who.int/classifications/apps/icd/icd10online/. Consulté le 27 avril 2010. En anglais.

28. Suivi du dossier sur les coûts non financés. Avastin. Montréal (QC) : Association québécoise d'établissements de santé et de services sociaux; 2008. Publié à www.aqesss.qc.ca/470/imedia.aspx?sortcode=1.1.3.4\&id=231. Consulté le 20 avril 2009.

29. Programme de gestion thérapeutique des médicaments (PGMT) [site web]. Programme de gestion thérapeutique des médicaments; 2009. Publié à www.pgtm.qc.ca. Consulté le 22 avril 2009.

30. Le Service canadien de prescription et d'utilisation optimales des médicaments. Ottawa $(\mathrm{ON})$ : Agence canadienne des medicaments et des technologies de la santé; 2009. Publié à http://cadth.ca/index.php/en/ compus. Consulté le 22 avril 2009. 
31. Observatoire des médicaments, des dispositifs médicaux et des innovations thérapeutiques de la région Centre (OMéDIT Centre) [site web]. Tours (France) : Cellule de Coordonation, OMéDIT Centre; 2009. Publié à www.omedit-centre.fr/. Consulté le 22 avril 2009.

32. Circulaire $n^{\circ}$ DSS/FSS/DHOS/E2/SG/2009/180 du 16 juin 2009 relative aux actions locales à conduire (contrôle des contrats de bon usage, application du dispositif de régulation) pour la maîtrise des produits de santé des listes en sus. Paris (France) : Gouvernement de la République française, Ministère de la Santé et des Sports; 2009.

Jean-François Bussières, B. Pharm., M. Sc., MBA, FCSHP, est chef du département de pharmacie et de l'unité de recherche en pratique pharmaceutique, CHU Sainte-Justine, et professeur titulaire de clinique, Faculté de pharmacie, Université de Montréal, Montréal, Québec.
Mariève Simoncelli, B. Pharm., est pharmacienne au département de pharmacie, CHU Sainte-Justine, Montréal, Québec.

Olivier Bourdon, D. Pharm., est Maître de conférences, Faculté de pharmacie, Université Paris-Descartes, et praticien hospitalier, AP-HP, Hôpital Robert-Debré, Paris, France.

Jean Lachaîne, Ph.D., est professeur agrégé à la Faculté de pharmacie, Université de Montréal, Montréal, Québec.

Adresse de correspondance :

Jean-François Bussières

Pharmacie

CHU Sainte-Justine

3175, chemin de la côte Sainte-Catherine

Montréal (Québec) H3T 1C5

courriel : jf.bussieres@ssss.gouv.qc.ca

\section{Reminder: the CJHP is now available online}

The Canadian Society of Hospital Pharmacists (CSHP) would like to remind readers about the online hosting platform now available for the Canadian Journal of Hospital Pharmacy (CJHP).

Our members and CJHP subscribers made it clear that an easy-to-use and fully searchable electronic version of the CJHP is important to them. The new CJHP website, launched in April 2009, uses Open Journal Systems (OJS) software to bring you each issue in a clear and readable electronic format. The intuitive OJS platform offers full searching of the CJHP archives that are available to date. Currently, the CJHP online has a complete archive of issues from 2000 to the present, with plans to add more issues from earlier volumes in the future.

CSHP recently announced it would be extending the open access to all of the CJHP archives and current issues for 2010! We encourage members and subscribers to spread the word about this free trial, especially to any colleagues who are not yet members of CSHP or who do not subscribe to the CJHP.

The new electronic CJHP is available at: www.cjhp-online.ca. All users will need to register to use the OJS system using the "Register" link in the top menu bar.

If you have any questions, please feel free to contact Colleen Drake, CSHP's Publications Administrator, by phone at 613-736-9733 ext. 228 or email at cdrake@cshp.ca. 